\title{
Repurposing Denosumab to Stabilize Acetabular Protrusio: Obviating Surgery
}

\author{
Sanjay Agarwala1, Mayank Vijayvargiya ${ }^{2}$ \\ ${ }^{1}$ Chief of Surgery and Director Professional Services, P. D. Hinduja Hospital and Medical Research Centre, Mumbai, India \\ ${ }^{2}$ Department of Orthopedics, P. D. Hinduja Hospital and Medical Research Centre, Mumbai, India \\ Email: drsa2011@gmail.com, maksy.doc37@gmail.com
}

How to cite this paper: Agarwala, S. and Vijayvargiya, M. (2020) Repurposing Denosumab to Stabilize Acetabular Protrusio: Obviating Surgery. Open Journal of Orthopedics, 10, 110-115.

https://doi.org/10.4236/ojo.2020.105012

Received: April 13, 2020

Accepted: May 10, 2020

Published: May 13, 2020

Copyright $\odot 2020$ by author(s) and Scientific Research Publishing Inc. This work is licensed under the Creative Commons Attribution International License (CC BY 4.0).

http://creativecommons.org/licenses/by/4.0/

(c) (i) Open Access

\begin{abstract}
Intrapelvic prosthetic migration prosthesis following hip arthroplasty can occur due to aseptic loosening, infection, injury and malposition of the cup with chronic instability. Revision surgery is the treatment option, but is often complex, is high risk owing to the co-morbidities of the patient, has higher complications and sometimes even patients refuse for the surgery. Osteoclast mediated bone resorption at the prosthetic bone interface is the main pathophysiology process involved in aseptic loosening associated intrapelvic migration. RANK/RANKL (Receptor Activated Nuclear factor $\kappa \mathrm{B}$ Ligand) is the primary pathway responsible for the periprosthetic osteolysis, therefore, we have offered Denosumab which binds to RANKL and inhibits osteoclasts mediated bone resorption, to our two patients with intrapelvic prosthetic migration who have refused for the revision surgery. Here, we report the outcome of these two cases of Intrapelvic prosthetic migration following a hip arthroplasty that was treated using subcutaneous injection of $120 \mathrm{mg}$ denosumab monthly for 3 months. Both the cases had good functional outcomes and radiographs showed good consolidation of bone around the prosthesis. These cases suggest denosumab can be repurposed to arrest further intrapelvic prosthetic migration due to its anti-resorptive and bone forming action and can avoid the need for a complex revision surgery.
\end{abstract}

\section{Keywords}

Denosumab, Re-Purpose, Prosthetic Migration, Osteolysis

\section{Introduction}

Intrapelvic prosthetic migration is a rare complication following hip replacement surgeries. Revision surgery has been the treatment option; however, it is complex and has higher complication rates [1]. With associated co-morbidities, patients opt 
not to consent for major surgery and/or are sometimes unfit for the surgery. Aseptic loosening is considered to be one of the main causes for intrapelvic prosthetic migration, where osteoclast mediated bone resorption is the pathology involved [2]. Macrophages phagocytose wear particles and release humoral factors which activate osteoblasts to stimulate osteoclastic bone resorption [3]. RANK/RANKL (Receptor Activated Nuclear factor $k \mathrm{~B}$ Ligand) is the primary pathway responsible for this periprosthetic osteolysis [4]. Thus, inhibiting osteoclast mediated bone resorption can play a role in preventing aseptic loosening and further protrusion.

Denosumab is a recombinant monoclonal antibody which binds to the RANKL and inhibits osteoclasts resulting in reduced bone resorption. Denosumab in 120 $\mathrm{mg}$ monthly doses has resulted in cessation of osteolysis, new bone deposition, redefinition of cortical margins and new woven bone formation in Giant cell tumor of bone (GCTB) [5]. Similar doses have also proved to be beneficial in the management of skeletal related events in metastatic tumours [6].

We have repurposed the use of Denosumab for the management of aseptic loosening associated intrapelvic prosthetic migration based on its role in inhibiting osteoclast mediated bone resorption and new bone formation. We share the results of two cases that were treated using Denosumab. Informed consent has been obtained from both the patients to share their radiographs and other details to be published.

\section{Case 1}

A 63-year-old lady presented with pain and inability to walk since 1 year. Patient underwent Total Hip Arthroplasty of the right hip 23 years ago with subsequent two revisions for aseptic loosening of the cup with the last revision done in 2013. Clinical and laboratory investigations ruled out infection. Radiographs showed intrapelvic migration of the acetabular cup (Figure 1).

In view of age related co-morbidities of DM, Hypertension, previous history of cardiac surgery, patient did not consent for the surgery and was therefore counselled for an empirical trial with subcutaneous injection of denosumab 120 mg monthly for 3 months after obtaining written informed consent. At 1 year follow-up, the patient was asymptomatic and was able to walk unaided. Her pain score (VAS) significantly reduced from 7 to 1 . Modified Harris Hip score (HHS) improved from 34 to 64 . Radiographs suggested good consolidation of bone around the acetabulum cup and stabilization of the protrusio.

\section{Case 2}

A 78-year-old lady presented to us 7 months after the bipolar hemiarthroplasty surgery with pain. Clinical and laboratory investigations ruled out infection. Radiographs suggested superior and medial migration of the prosthesis (Figure 2). Considering the co-morbidities like DM, hypertension, Rheumatoid Arthritis and asthma, patient did not consent for a revision surgery. The patient was counselled for an empirical trial with subcutaneous injection of Denosumab $120 \mathrm{mg}$ monthly for 3 months after obtaining written informed consent. 

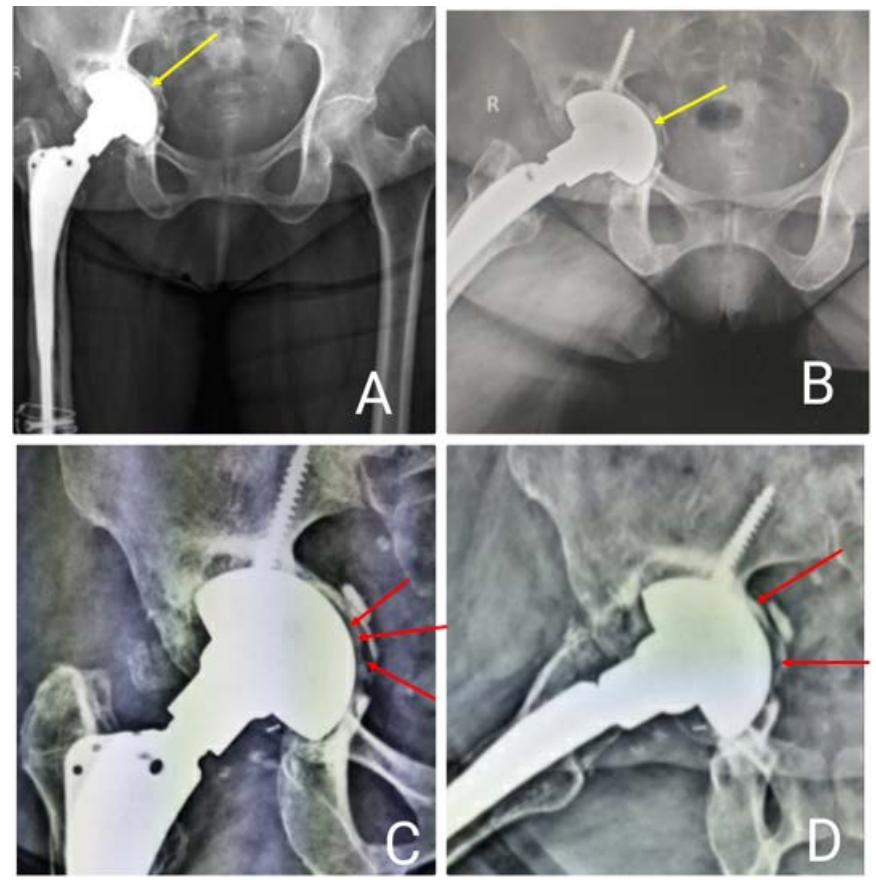

Figure 1. ((A), (B)) Anteroposterior and lateral radiographs showing intrapelvic migration of prosthesis (yellow arrow) in a 63 years old lady following a revision total hip replacement. ((C), (D)) Anteroposterior and lateral radiographs after 1 year follow up showing the arrest of further protrusion with formation of a bony shell with consolidation around the acetabular cup (red arrow).

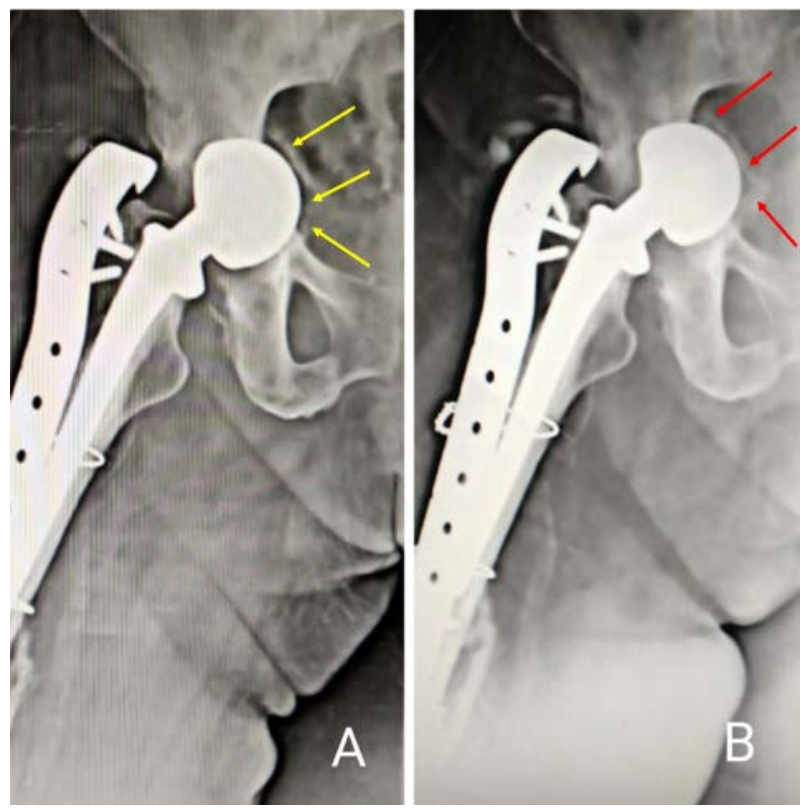

Figure 2. (A) Anteroposterior radiograph of a 78 years old lady showing protrusion of the bipolar prosthesis (yellow arrow). (B) Anteroposterior radiograph after 1year follow up with denosumab therapy showing arrest of further protrusion with formation of a bony shell around the head of prosthesis (red arrow). 
At the end of 6 months of this therapy, the patient was asymptomatic and could walk unaided. Her pain score (VAS) improved from 6 to 2. The modified HHS improved from 36 to 62 . At last follow-up visit at 15 months, patient was asymptomatic and was walking unaided. The clinical findings could be correlated with the radiological findings that suggested stabilization of the protrusio and good bone consolidation around the prosthetic head.

\section{Discussion}

Aseptic loosening is the main pathophysiology associated with intrapelvic prosthetic migration where osteolysis at the implant bone interface is often the first step involved. Particulate wear debris generated at the articular bearing surface and at other non-articular prosthesis or cement surfaces initiates a foreign body reaction characterized by granulomas that are rich in macrophages and multinucleated giant cells [3].

These macrophages in-turn stimulate osteoclasts by two mechanisms: 1) produce humoral factors like TNF, IL-1, RANKL that activate osteoblasts which in turn stimulate osteoclast mediated bone resorption, 2) differentiate into bone resorbing osteoclasts contributing directly to osteolysis [7]. Osteoclast differentiation is primarily controlled by the stromal/osteoblast-derived proteins RANKL and macrophage colony-stimulating factor. The effect of TNF on stimulation and activation of osteoclastogenesis appears to be entirely dependent on an active RANK/RANKL pathway [8]. Thus the RANK/RANKL is the principal axis that regulates periprosthetic osteolysis [8]. Any treatment which can inhibit osteoclast mediated bone resorption can inhibit osteolysis and can thus prevent or arrest osteolysis related intrapelvic migration.

RANKL antibody/antagonist, such as denosumab prevents RANKL from binding to the RANK receptors on the surface of osteoclasts thereby, inhibiting osteoclastogenesis. Kostenuik et al. in an animal study concluded that Denosumab inhibits bone resorption and increases bone mass density [9].

Denosumab has been used in GCTB and in treatment of skeletal related bone events in metastatic cases owing to its osteoclastogenesis properties. In GCTB, when given $120 \mathrm{mg}$ subcutaneously every monthly, Denosumab has proved to be beneficial in the cessation of osteolysis, new woven bone formation, redefinition of ill-defined cortical margins and new bone deposition [5]. It has also been effectively used for delaying and prevention of skeletal related events in metastatic cases in the same dosage [6]. Denosumab has also been shown to increase callus volume, density and delays callus remodeling in fracture healing [10]. Thus, these studies in three different conditions have proven the role of Denosumab in inhibiting bone resorption and in promoting new woven bone formation.

In our study, we have used the same principle of Denosumab in cases of aseptic loosening associated intrapelvic prosthetic migration. When given in $120 \mathrm{mg}$ subcutaneous dosage monthly for 3 months, it has resulted in cessation of osteolysis, prevented further migration of the prosthesis and stabilized the protru- 
sio by formation of new bone around the prosthesis. This was very well reciprocated clinically, where patients who were unable to bear weight became asymptomatic and could walk unaided. Radiologically, the protrusio stabilized with consolidation of new bone around the prosthesis. Based on the review of literature, there is enough evidence to explain the effect of Denosumab in our study to arrest and prevent osteolysis and promote new bone formation in aseptic loosening associated intrapelvic prosthetic migration. However, large case series/randomised control trials would be needed to support our findings.

\section{Conclusion}

Our study concludes that Denosumab due to its anti-osteoclastic properties have a role in the non-surgical management of aseptic loosening associated intrapelvic migration. Denosumab can be repurposed to arrest further intrapelvic prosthetic migration due to its anti-resorptive and bone forming action and can avoid the need for a complex revision surgery.

\section{Conflicts of Interest}

The authors declare no conflicts of interest regarding the publication of this paper.

\section{References}

[1] Bach, C.M., Steingruber, I.E., Ogon, M., Maurer, H., Nogler, M. and Wimmer, C. (2002) Intrapelvic Complications after Total Hip Arthroplasty Failure. The American Journal of Surgery, 183, 75-79. https://doi.org/10.1016/S0002-9610(01)00845-5

[2] Goodman, S.B., Chris, R.C., Chiou, S.S., Schurman, D.J., Woolson, S.T. and Masada, M.P. (1989) A Clinical-Pathological-Biochemical Study of the Membrane Surrounding Loosened and Nonloosened Total Hip Arthroplasties. Clinical Orthopaedics and Related, 244, 182-187. https://doi.org/10.1097/00003086-198907000-00017

[3] Murray, D.W. and Rushton, N. (1990) Macrophages Stimulate Bone Resorption When They Phagocytose Particles. The Journal of Bone and Joint Surgery, 72B, 988-992. ttps://doi.org/10.1302/0301-620X.72B6.2246303

[4] Childs, L., Paschalis, E., Xing, L., Dougall, W., Anderson, D., Bosky, A., et al. (2002) In Vivo RANK Signaling Blockade Using the Receptor Activator of NF- $\kappa$ B:Fc Effectively Prevents and Ameliorates Wear Debris-Induced Osteolysis via Osteoclast Depletion without Inhibiting Osteogenesis. Journal of Bone and Mineral Research, 17, 192-199. https://doi.org/10.1359/jbmr.2002.17.2.192

[5] Branstetter, D.G., Nelson, S.D., Manivel, J.C., et al. (2012) Denosumab Induces Tumor Reduction and Bone Formation in Patients with Giant-Cell Tumor of Bone. Clinical Cancer Research, 18, 4415-4424.

https://doi.org/10.1158/1078-0432.CCR-12-0578

[6] Stopeck, A.T., Lipton, A., Body, J.J., et al. (2010) Denosumab Compared with Zoledronic Acid for the Treatment of Bone Metastases in Patients with Advanced Breast Cancer: A Randomized, Double-Blind Study. Journal of Clinical Oncology, 28, 5132-5139. https://doi.org/10.1200/JCO.2010.29.7101

[7] Sabokbar, A., Fujikawa, Y., Neale, S., Murray, D.W. and Athanasou, N.A. (1997) Human Arthroplasty Derived Macrophages Differentiate into Osteoclastic Bone Re- 
sorbing Cells. Annals of the Rheumatic Diseases, 56, 414-420.

https://doi.org/10.1136/ard.56.7.414

[8] Zhang, Y., Heulsmann, A., Tondravi, M., Mukherjee, A. and Abu-Amer, Y. (2001) Tumor Necrosis Factor-Alpha (TNF) Stimulates RANKL-Induced Osteoclastogenesis via Coupling of TNF Type I Receptor and RANK Signaling Pathway. Journal of Biological Chemistry, 276, 563-568. https://doi.org/10.1074/jbc.M008198200

[9] Kostenuik, P.J., Nguyen, H.Q., McCabe, J., Warmington, K.S., Kurahara, C., Sun, N., et al. (2008) Denosumab, a Fully Human Monoclonal Antibody to RANKL, Inhibits Bone Resorption and Increases BMD in Knock-In Mice That Express Chimeric (Murine/Human) RANKL. Journal of Bone and Mineral Research, 24, 182-195. https://doi.org/10.1359/jbmr.081112

[10] Hegde, V., Jo, J.E., Andreopoulou, P. and Lane, J.M. (2016) Effect of Osteoporosis Medications on Fracture Healing. Osteoporosis International, 27, 861-871.

https://doi.org/10.1007/s00198-015-3331-7 